\title{
Elimination of Tec elements involves a novel excision process
}

\author{
John W. Jaraczewski ${ }^{1,2}$ and Carolyn L. Jahn ${ }^{1,3}$ \\ ${ }^{1}$ Department of Cell, Molecular, and Structural Biology, Northwestern University Medical School, Chicago, \\ Illinois $60611 \mathrm{USA}^{2}{ }^{2}$ Department of Biological Sciences, University of Illinois at Chicago, Chicago, Illinois 60680 USA
}

\begin{abstract}
Approximately 60,000 transposon-like elements of the Tec1 and Tec2 families excise en masse from the micronuclear genome during formation of a macronucleus in Euplotes crassus. The circular product has been shown previously to contain the element inverted repeats joined head to head. To elucidate the mechanism of Tec excision, we have further characterized the circular products. DNA sequence analysis of cloned inverted repeat junctions and of a population of supercoiled Tec circles shows that the inverted repeat junctions consist of both copies of the target site duplication surrounding 10 additional bases. The 10 bp differs for each junction. We demonstrate that the circles are highly sensitive to $S 1$, mung bean and Bal 31 nucleases, and the site of sensitivity maps to the junction. Alkaline gel electrophoresis indicates that the junction does not contain a nick or gap; thus, a likely explanation for the nuclease sensitivity is the existence of a heteroduplex DNA structure at the junction. On the basis of these results, we present a model of Tec excision and discuss the relationship of Tec excision to IES elimination and chromosome fragmentation in E. crassus.
\end{abstract}

[Key Words: Extrachromosomal circle; ciliated protozoa; transposon; DNA rearrangement]

Received September 10, 1992; revised version accepted November 9, 1992.

Hypotrichous ciliated protozoa eliminate massive amounts of DNA during the formation of a macronucleus from a micronucleus (for review, see Klobutcher and Prescott 1987; Jahn 1991; Klobutcher and Jahn 1991). These two nuclei serve different functions that allow a dramatic difference in sequence complexities. The macronucleus is a transcriptionally active, somatic nucleus that is destroyed and regenerated during the sexual phase of the ciliate life cycle. The micronucleus is an inactive germ-line repository of genetic information that divides meiotically producing haploid products that participate in sexual exchange between conjugating cells. A new macronucleus is generated from a copy of a zygotic micronucleus formed after fusion of haploid micronuclei. In hypotrichous ciliates, $\sim 95 \%$ of the micronuclear genome is eliminated in the process of forming the macronuclear genome, which is comprised of $\sim 24,000$ short, linear DNA molecules, each bearing a gene and terminating in simple telomeric repeat sequences. DNA elimination is required for the constitution of functional genes in the macronucleus; thus, the DNA elimination process resembles other developmentally regulated gene rearrangements. The ciliate rearrangements differ in at least one respect-their abundance-and as many as $10^{5}$ rearrangements occur within a single nucleus.

DNA elimination by DNA breakage and reunion has been described for several ciliates (Yao 1989; Klobutcher

\footnotetext{
${ }^{3}$ Corresponding author.
}

and Jahn 1991). In Euplotes crassus, two types of DNA breakage and reunion processes have been characterized extensively. One is the elimination of two related transposon-like element families, Tec1 and Tec2 (Baird et al. 1989; Jahn et al. 1988,1989; Krikau and Jahn 1991; Tausta et al. 1991). These elements are $\sim 5.3 \mathrm{~kb}$ in length with 700 to 725 -bp terminal inverted repeats. Each element is present in $\sim 30,000$ copies, and as many as $50 \%$ of the elements interrupt macronuclear-destined sequences. During a 4-hr time period of macronuclear development, all of the Tec elements are excised from the genome. A second process involves the elimination of IESs (internal eliminated sequences): These are short (31-374 bp) unique sequence interruptions of the macronuclear-destined sequences (Baird et al. 1989; Tausta and Klobutcher 1989,1990; Tausta et al. 1991). At least 10,000 IESs must be excised during macronuclear development in E. crassus. There are striking similarities between these elimination events. Both processes result in the formation of a circular product from the excised DNA; the structure of the circles is similar, and elimination is highly precise. Both Tec element and IESs are bounded by a short (2-4 bp) duplication of sequence that resembles a target site duplication; and in both cases, the macronuclear-destined products of excision are precisely rejoined such that only one copy of this duplication is retained in the macronuclear product of the excision. Sequence analysis of one Tecl circular product indicated that the junction formed between the inverted repeats 
contained both copies of the target site duplication surrounding 10 extra bp. Similarly, all of the IES circle junctions that have been sequenced retain both copies of the duplication with extra base pairs in between, typically $10 \mathrm{bp}$. Because IESs are unique sequences and their integrated and circular forms have been sequenced, it is clear that the extra base pairs at the junction of the excised circles are derived from sequences that surround the IES in the precursor to the macronuclear-destined sequence. These data suggest that a staggered cut is made surrounding the IES: This would allow the ends to be filled in and precisely rejoined to generate the macronucleardestined sequence and could generate the three copies of the target site duplication /one in the macronuclear-destined sequence and two in the circular IES). A similar mechanism could give rise to the Tec circle junctions, as these also give rise to precisely rejoined macronuclear sequences.

Despite the above similarities, the developmental timing of these elimination events differs (Jahn et al. 1989; Tausta and Klobutcher 1989,1990; Krikau and Jahn 1991). The excision of Tec elements occurs early in the formation of a macronucleus, as micronuclear chromosomes become polytenized. The IES removal occurs $15-$ $25 \mathrm{hr}$ later at the end of polytenization and just before a major DNA degradative stage that coincides with formation of the small linear DNA molecules. Thus, any models of excision of Tecs and IESs must take into consideration additional factors that alter the timing of these events.

Circular forms of excised DNA have been found in several other sequence-specific DNA rearrangement processes as either an intermediate or end product. In prokaryotes, circular products have been observed for Tn10 and Tn916. Two types of circular structures are formed by Tn10 transposase (Morisato and Kleckner 1987; Haniford et al. 1991). One is a noncovalently closed structure that consists of a protein-DNA complex formed between the transposase and the ends of the element (called the ETF, or excised transposon fragment). This structure is closely related to intermediates formed by phage Mu during transposition and may be common to all transposition mechanisms (Haniford et al. 1991; Mizuuchi 1992). The second structure is a circle formed by transposition of the element into itself. Although neither of these $\operatorname{Tn} 10$ circles resembles the structures seen in E. crassus, their presence as intermediates and products of $\operatorname{Tn} 10$ transposition point to the mechanistic role of circularization in DNA rearrangements. In contrast to Tn10 or Mu transposition, the Tn916 conjugative transposon generates a covalently closed circular intermediate (Caparon and Scott 1989). The recombination proteins encoded by Tn916 resemble phage $\lambda$ Int and Xis; thus, the transposition mechanism appears to be related to the $\lambda$-type site-specific recombination (Poyart-Salmeron et al. 1989, 1990). The junction of the transposon termini in the Tn916 circular intermediate is a heteroduplex that contains extra nucleotides derived from adjacent chromosomal sequences from each end of the integration site. This circular product is capable of reinte- gration (Scott et al. 1988). The Tn916 transposon is unusual in that it does not create a target site duplication. Instead, it is proposed that the sequences found in the circular form heteroduplex junction provide "coupling sequences" that are cut via staggered nicks and ligated into a similarly cut target sequence. The existence of this staggered cut and the heteroduplex structure is highly relevant to the structure of the E. crassus IES and Tec circular structures described above and provides a precedent for our interpretation of the results presented below.

In eukaryotes, circular forms of the Caenorhabditis elegans Tcl element and the Zea mays Mul elements have been detected (Rose and Snutch 1984; Ruan and Emmons 1984; Sundaresan and Freeling 1987). Like the Tec element circles in E. crassus, the Tcl and Mul inverted repeats are joined in a head-to-head orientation in the circles. In the Tcl circle, the inverted repeat junction seems to be precise. The junction can be digested with $R s a I$ as predicted from the DNA sequence data if the junction consists of a single copy of the target site duplication in between head-to-head inverted repeats. Studies of both Tcl and Mul have concentrated on the excision site, or footprint, left behind and not on the circular product (Moerman and Waterston 1989; Doseff et al. 1991). This has demonstrated that excision is generally imprecise for these transposons and, thus, differs from what is seen in E. crassus. To date, there is no direct evidence for the reinsertion of the excised circular transposons $\mathrm{Tcl}$ and $\mathrm{Mul}$, although the frequency of transposition correlates with the presence of circular forms.

More detailed understanding of the relationship of circular structures to a recombination mechanism in a eukaryote comes from the analysis of circular recombination products generated from deleted DNA during immunoglobulin and $\mathrm{T}$-cell receptor gene rearrangements (Fujimoto and Yamagishi 1987; Okazaki et al. 1987; McCormack et al. 1989). Circular products of $V-D-I$ joining for both types of genes indicate that heptamer sequences are joined in a head-to-head orientation. These heptamer-heptamer products are precisely fused or, in some cases (Okazaki et al. 1987), retain a few base pairs that appear to be derived from adjacent germ-line sequence. These structures lend support to an intramolecular deletion model of $V \cdot D-J$ joining involving precise cutting near heptamer sequences and heptamer-heptamer joining. The precision of heptamer-heptamer joining in the circular products contrasts with the heterogeneity of coding junctions and thus points to mechanistic differences in the processing of the two types of ends.

To gain insight into the mechanism of excision of Tec elements, we have undertaken a detailed study of the Tec element termini and their junction in the circular Tec excision product. The results presented here indicate the presence of $10 \mathrm{bp}$ between the inverted repeats at the circular junction. We also report that an unual nuclease-sensitive conformation exists at the circular junction that may be the result of the presence of heteroduplex DNA. 


\section{Results}

\section{Tec element inverted repeat consensus sequence}

In the process of identifying the end points of Tec elements in micronuclear phage clones and in characterizing the circular forms of the elements, we have sequenced the first $60-70 \mathrm{bp}$ of 17 copies of the Tec1 inverted repeat and 9 copies of the Tec2 element inverted repeat. Alignment of these sequences generates the consensus sequences shown in Figure 1. Because gaps are required to align the sequences from $\mathrm{Tec} 2$ with those from Tecl (see legend to Fig. 1), the consensus for each element is also shown. Two regions show noticeable conservation between all of the elements: (1) the first 7 nucleotides, and (2) the sequence $5^{\prime}$-ATA-TTGAA-AC$3^{\prime}$, beginning $14 \mathrm{bp}$ from the $5^{\prime}$ end of the inverted repeat. The TTGAA sequence motif (2, above) forms part of a larger conserved sequence that closely resembles a putative consensus sequence for chromosome fragmentation and telomere addition (Baird and Klobutcher 1989). As a chromosome fragmentation consensus sequence, the $5^{\prime}$ TTGAA-3' is located 17 bp away from the fragmentation site (Fig. 1B). This is the same distance found for the TTGAA motif in the inverted repeat ends of Tec elements relative to the center of the TA target site duplication (Fig. 1B). A second copy of the TTGAA motif is present beginning 52 and $53 \mathrm{bp}$ from the $5^{\prime}$ end of the inverted repeats of Tecl and Tec2, respectively (see individual Tec1 or Tec2 consensus in Fig. 1A).

\section{Cloning and sequencing extrachromosomal Tec inverted repeat junctions}

During our initial characterization of circular Tec elements, we showed that uniquely sized restriction fragments containing the inverted repeats are produced from the circles and correspond to the size predicted if the inverted repeats are joined in a head-to-head orientation (Jahn et al. 1989; Krikau and Jahn 1991). These fragments are a 2.9-kb EcoRI (illustrated in Fig. 2) and a 1.1-kb PstI fragment for Tecl and a $2.5-\mathrm{kb}$ HindIII-EcoRI, a $3.3-\mathrm{kb}$ HindIII, and a $1.3 \mathrm{~kb} \mathrm{XbaI}$ fragment for Tec2. In addition, we cloned an extrachromosomal circular form of Tecl. This clone, IR13, contained the characteristic $2.9-\mathrm{kb}$ EcoRI restriction fragment specific for excised, circular Tec1. Sequence analysis confirmed that the inverted repeats were joined in a head-to-head orientation and demonstrated that 10 extra bp existed between the ends of the inverted repeats. To determine whether the sequence of Tecl circle junctions is a particular sequence or a defined number of bases, we cloned and sequenced five more inverted repeat junctions from excised Tecl and two from excised Tec2.

Tecl junctions were cloned from anlagen (developing macronuclear) DNA as 2.9-kb EcoRI fragments or as 1.1$\mathrm{kb}$ Pst fragments from gel-purified circular forms (see Materials and methods). Tec2 junctions were cloned as 2.5-kb HindIII-EcoRI fragments from gel-purified circular forms. Positive clones were identified by hybridization using probes specific for the inverted repeats of Tecl or Tec2. The number of clones identified were fewer than expected, presumably owing to the instability of 700-bp inverted repeats in Escherichia coli (Collins et al. 1982). Sequencing through the junction of the inverted repeats from each clone (Fig. $1 \mathrm{C}$ ) revealed 10 extra nucleotides in all cases. These 10 bases share no obvious sequence or structural identity with each other. The inverted repeats in two of the junction clones have deletions (pJ2 and $\mathrm{pJ} 12$ ) or additions (pJ2) relative to the consensus sequence. In addition, three pairs of inverted repeats in the junctions (pJ2, pJ8, and pANL31) are $<81 \%$ identical in sequence for the region analyzed, and only two inverted repeat pairs in the cloned junctions (pJ34 and pJ46) are $>96 \%$ identical. This is in striking contrast to the inverted repeat pairs that we have sequenced from integrated copies of Tec elements: Two of these elements showed $100 \%$ identity of their inverted repeats, and two others are $91 \%$ and $98 \%$ identical (Jahn et al. 1989; Krikau and Jahn 1991; M. Krikau, J. Frels, and C. Jahn, unpubl.). This suggests that cloning of the junctions results in a bias toward junctions containing inverted repeats that have decreased sequence similarity: These are probably more stable in $E$. coli.

We also analyzed the bases present at the junction of the inverted repeat by sequencing a population of extrachromosomal circular Tecs. Supercoiled Tec circles were purified from total DNA samples isolated at various times of macronuclear development using $\mathrm{CsCl} /$ ethidium bromide gradients. Sequence across the junction of circular Tecls was obtained using a 37-bp Tec1-specific oligonucleotide primer that anneals within both inverted repeats (on opposite strands) at a position $68 \mathrm{bp}$ from the junction. The sequence revealed a particular base at all conserved positions, a 2-base stop at positions utilizing either of 2-bases, and a 4-base stop at positions utilizing any base (Fig. 3). We can conclude from this analysis that the junction of many of the extrachromosomal Tecl circles consists of the following sequence: ACTCCCTCTATANNNNNNNNNNTATAGAGGGAGT (the TA target site duplication is underlined.)

\section{Nuclease sensitivity of circular forms of Tec}

Although it is possible to obtain DNA preparations containing only supercoiled circular forms of Tec elements, many preparations contain nicked circles and linears, particularly when the DNA was prepared from purified anlagen (Jahn et al. 1989). We have shown previously that the linears are the result of random endonuclease attack during preparation of the anlagen DNA. To further assess the conformation of the circular Tecs, we reacted total DNA (including macronuclear, micronuclear, and anlagen DNA/ with $\mathrm{S} 1, \mathrm{Bal} 31$, or mung bean nuclease followed by restriction enzyme digestion to determine whether a specific region of the circle preferentially reacts with the nucleases. Specifically, 30-hr total DNA / $30 \mathrm{hr}$ refers to the postmixing time of two mating types and corresponds to $\sim 6 \mathrm{hr}$ of macronuclear development) was allowed to react with the different nu- 
A

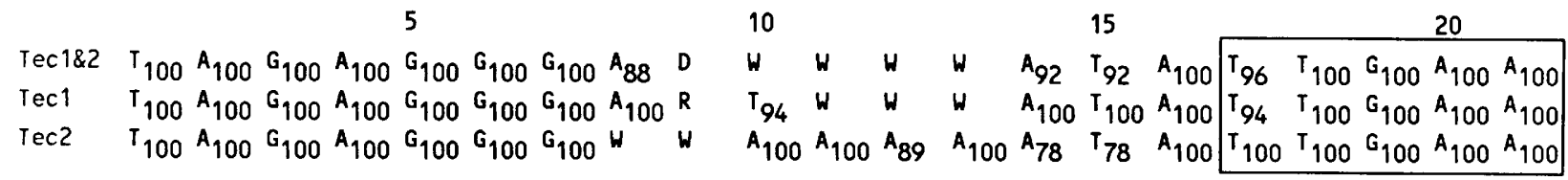

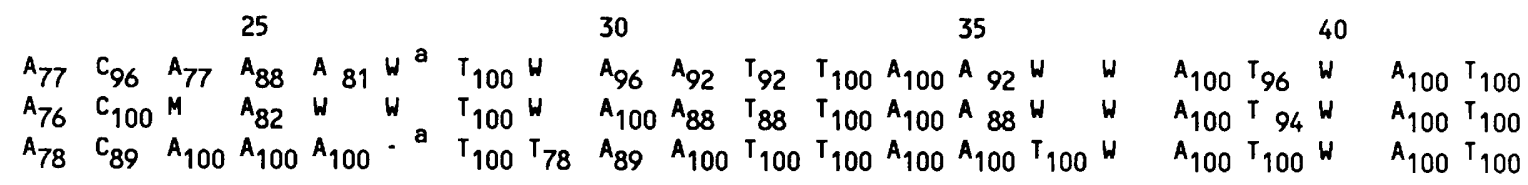

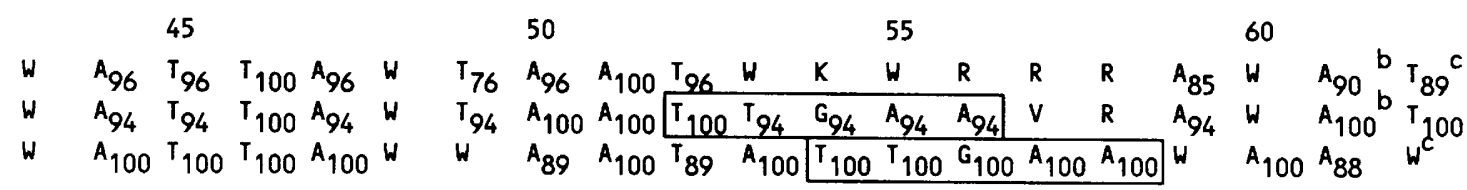

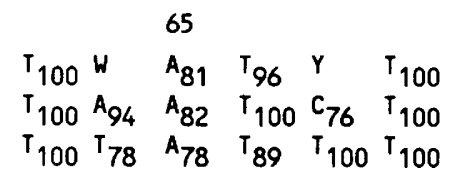

a represents $16 / 17$ Tec 1,0 Tec 2 sequences

b represents $2 / 17$ Tec1, 8/9 Tec2 sequences

c represents 15/17 Tec1, 4/9 Tec2 sequences

$D=A / G / T$

$W=A / T \quad M=A / C$

$K=G / T \quad R=A / G$

$Y=C / T \quad V=A / C / G$

B

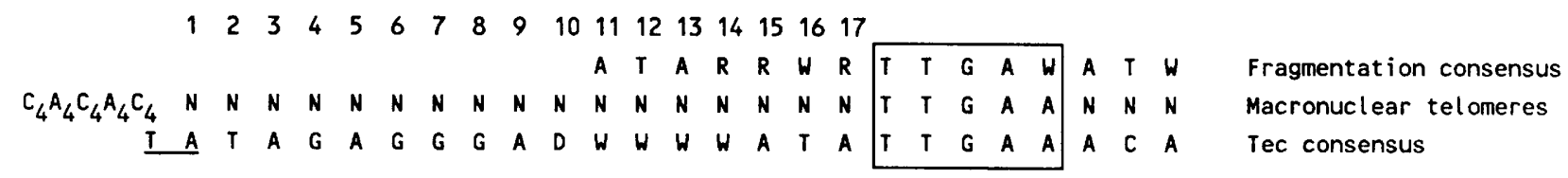

C

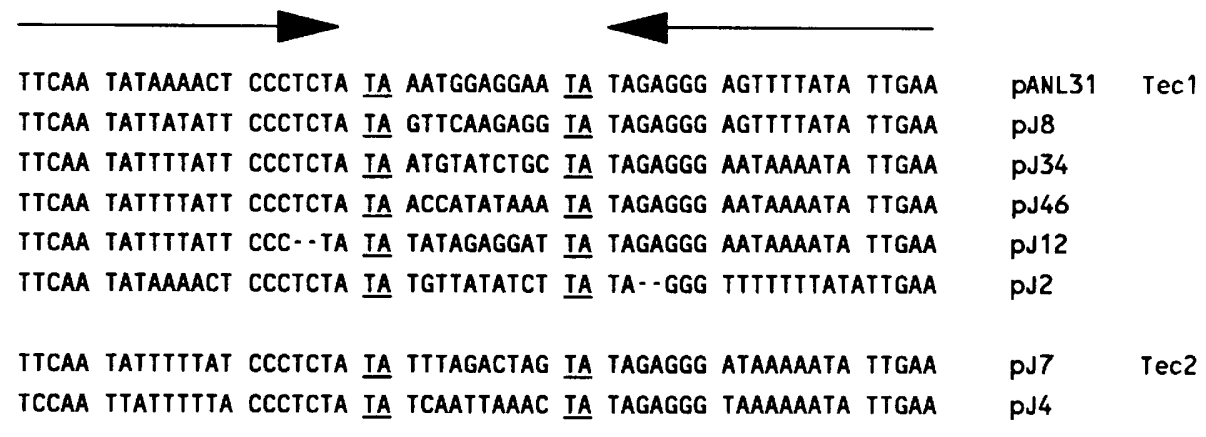

Figure 1. DNA sequence of Tec element inverted repeats and circular form junctions. (A) The PILEUP program of the UWGCG system (Devereux et al. 1984) was used to align $17 \mathrm{Tec} 1$ and $9 \mathrm{Tec} 2$ inverted repeat sequences, and the CONSENSUS program was then used to determine the frequencies of each nucleotide at each position. Results are shown for alignments of all of the sequences (Tec1\&2) and for Tecl vs. Tec2 sequences. Gaps introduced to align the sequences are indicated by the dash at position 27 , where, as noted by superscript $a$, only Tec1 sequences have a base at this position (all Tec2 sequences were gapped), as indicated 15 Tec1 sequences were gapped at the position noted by superscript $b$, and 2 Tecl and 5 Tec 2 sequences were gapped at the position noted by superscript $c$. The subscript numbers indicate the percentage of sequences containing that nucleotide. Residues with a single base representing $<70 \%$ of the sequences are given designations of $\mathrm{R}, \mathrm{W}, \mathrm{Y}$, etc. The alignment starts at base 1 of the element sequences. The numbering above the sequences refers to the Tec $1 \& 2$ consensus and the Tecl consensus. The Tec 2 consensus differs in numbering because of the gap introduced at the position noted by superscript $a$. The conserved TTGAA motifs are boxed. (B) The Tec element inverted repeat consensus is aligned with the sequence immediately internal to the macronuclear telomere (Klobutcher et al. 1981) and the chromosome fragmentation consensus sequence (Baird and Klobutcher 1989) to show the conserved TTGAA core consensus alignment. The underlined TA in the Tec sequence corresponds to the target site duplication. The $5^{\prime}$ end of the macronuclear telomere sequence shows the end of the $\mathrm{C}_{4} \mathrm{~A}_{4}$ repeats. The TTGAA consensus core is boxed. The numbering indicates the position of the TTGAA relative to the telomere in the macronuclear chromosome ends $\left(C_{4} A_{4}\right.$ repeats $)$. The fragmentation consensus occurs at this position in the macronuclear-destined sequence or can occur in the flanking eliminated sequence (not shown). (C) The sequence across the junction from six Tecl clones and two Tec 2 clones is shown. Each junction contains 10 extra nucleotides inserted between the target site duplications and inverted repeats. The arrows correspond to the inverted repeat sequence, and the TA target site duplication is underlined. The pANL 31 sequence was originally reported by Jahn et al. (1989). The derivation of pJ8, pJ34, pJ46, pJ12, pJ2, pJ7, and p/4 is described in Materials and methods. 


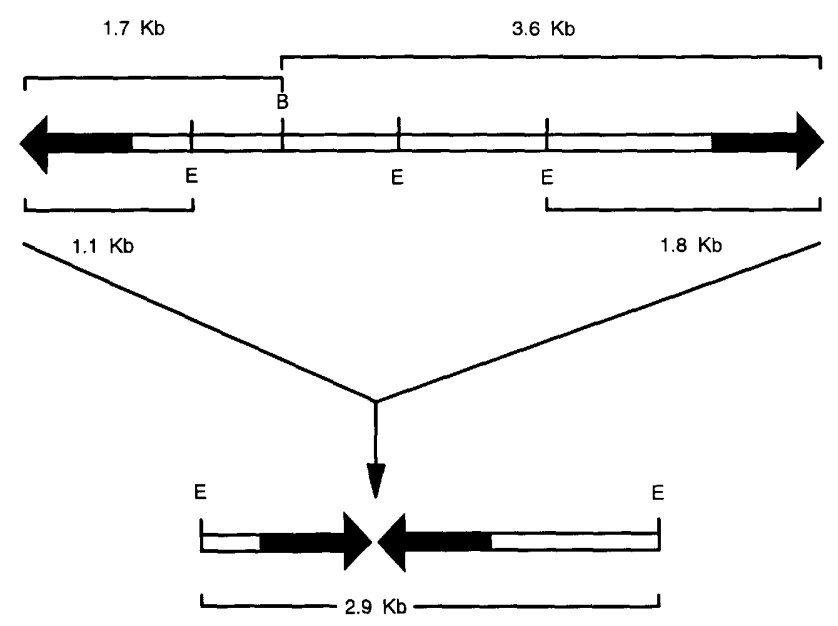

Figure 2. Maps of Tecl. The restriction maps of the most prevalent version of Tec1 in its integrated form (top) and a partial map of its excised circular form (bottom) are shown. The solid arrowheads represent the long terminal inverted repeats. The approximate distances from the ends of each inverted repeat to the internal EcoRI (E) and $B a m H I(B)$ restriction sites are given. Upon excision, the inverted repeats are joined together in a head-to-head orientation, creating a $2.9-\mathrm{kb}$ EcoRI fragment characteristic of the circle.

cleases for increasing periods of time. After phenol-extracting the DNA to stop the reactions, the DNA was electrophoresed in agarose gels, Southern blotted, and hybridized with a Tec-specific probe.

The Tecl S1 nuclease results can be seen in Figure 4A. The Southern blots have been probed with a $2.9-\mathrm{kb}$ EcoRI fragment Tec1 junction clone. Lanes 1 and 2 compare 30-hr total DNA, native and S1 digested for $15 \mathrm{~min}$, respectively. The majority of the circle is linearized by S1. To map the site of linearization, the 30-hr total DNA with or without prior S1 treatment was digested with either EcoRI or BamHI (lanes 3-6). EcoRI digestion results in the characteristic $2.9-\mathrm{kb}$ Tecl junction fragment. Prior S1 treatment reveals new 1.8 - and $1.1-\mathrm{kb}$ bands replacing the $2.9-\mathrm{kb}$ band. Because there is only a single BamHI restriction site in Tecl, BamHI digestion results in the linearization of the circle. Prior S1 treatment results in the appearance of 3.6 - and 1.7-kb fragments. Taken together, the site of $\mathrm{Sl}$ reactivity in the circle is at the inverted repeat junction (for mapping data, see Fig. 2). This was further verified by stripping the Southern blots and reprobing them with labeled fragments that distinguish between each end of the element: In each case, a fragment of the predicted size hybridized (data not shown). Substituting mung bean nuclease for S1 resulted in less reactivity but identical specificity /data not shown).

Bal 31 nuclease reacted very rapidly at the inverted repeat junction (Fig. 4B). At 1 min digestion time, linear Tecls can be seen (lane 2). By $5 \mathrm{~min}$, the double-strand exonuclease activity has shortened the linearized circle by reacting with the ends. BamHI digestion of DNA after $1 \mathrm{~min}$ of digestion with Bal 31 results in the appearance of the 3.6- and 1.7-kb fragments signifying a specificity for the inverted repeat junction as well. After $5 \mathrm{~min}$ of treatment with $\mathrm{Bal} 31, \mathrm{BamHI}$ digestion reveals that the exonuclease has reacted in both directions from the initial cleavage site because both the $3.6-$ and $1.7-\mathrm{kb}$ fragments are shorter.

The above experiments with S1 nuclease, mung bean nuclease and $\mathrm{Bal} 31$ have all been repeated with restriction enzyme digests and probes specific for the Tec2 element. We found that all three nucleases also specifically cleave at the inverted repeat junction of the Tec2 circle (data not shown). These three nucleases have similar but different sensitivities (Gray et al. 1975; Shenk et al. 1975; Wiegand et al. 1975; Kroeker and Kowalski 1978). All three act as single-stranded nucleases and as endonucleases at gaps, heteroduplex regions, or regions of unusual DNA structure (non-B DNA). Because Bal 31 and S1 nuclease cut inefficiently opposite nicks and mung bean nuclease does not cut opposite a nick, the data suggests that a several-base gap, non-B DNA conformation, or heteroduplex, exists at the junction of the inverted repeats of excised Tecs.

To examine the role of DNA conformation, the reactivity of the nucleases was also tested using one of the 2.9-kb EcoRI junction clones. This plasmid clone is expected to be in the same sequence-specific conformation (owing to the head-to-head 700 bp inverted repeats) as excised Tecs. This clone does not react specifically at the inverted repeat junction with any of the above nucleases. Plasmids that contain short inverted repeats have been shown to adopt a non-B DNA conformation when supercoiled, making them sensitive to S1 nuclease (Lilley 1980). Therefore, we tested for a conformational effect by first digesting the circular forms with restriction en-

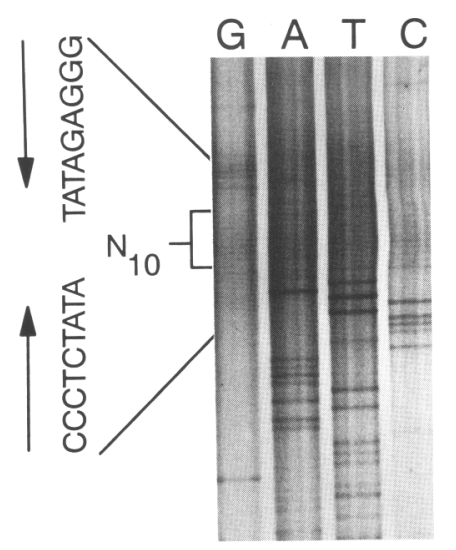

Figure 3. Junction sequence of purified Tecl circles. Supercoiled Tec circles were purified from total DNA by centrifugation in $\mathrm{CsCl} /$ ethidium bromide. The junction of the inverted repeats was sequenced using a primer that anneals within the Tec1 inverted repeat. Thus, sequence is obtained from both strands of the circles extending from the primer into the junction. The arrows designate the head-to-head inverted repeats. The junction is apparent as stops in every lane for a stretch of 10 nucleotides (shown as $\mathrm{N}_{10}$ ) in between the two copies of the target site duplication (TA). 
Figure 4. Nuclease sensitivity of circular Tecl. (A) Total DNA from cells at different times of macronuclear development was reacted with S1 nuclease for either 0 ( $\mathrm{S} 1$ buffer only) or $15 \mathrm{~min}$. The DNA was electrophoresed either with or without prior restriction enzyme digestion, Southern blotted, and probed with a clone containing a $2.9-\mathrm{kb}$ EcoRI junction fragment specific for Tecl. Native DNA (lane 1) contains excised Tecl circle visualized as a single band appearing to be $9-\mathrm{kb}$ in size. After S1 treatment for 15 min (lane 2), the majority of the circle has been linearized and now migrates at $5.3 \mathrm{~kb}$ in size. To map the location of the linearization, the S1-treated sample was digested with EcoRI or BamHI. EcoRI digestion of untreated DNA reveals the $2.9-\mathrm{kb}$ fragment characteristic of the circle (lane 3). All other bands present in this lane are the result of other versions of Tecl, polymorphic for EcoRI sites. EcoRI digestion after S1 treatment (lane 4) reveals the appearance of two additional bands, 1.8 and $1.1 \mathrm{~kb}$ in size. Because there is a single BamHI site in Tecl, BamHI digestion of circular Tecl DNA results in the linearization of the circle (lane 5). S1 treatment before BamHI digestion also results in the appearance of two new bands, 3.6 and $1.7 \mathrm{~kb}$ in size. The additional bands for both the S1-EcoRI and S1-BamHI experiments correspond to the distance that these restriction sites are located from the ends of the inverted repeats (Fig. 2), indicating that S1 nuclease treatment has linearized the circle at the junction of the inverted repeats. We have also noted a hybridizing band migrating above the limit-mobility micronuclear DNA in lane 1. With S1 treatment, this migrates as $\sim 10.6 \mathrm{~kb}$ in size, which is the size expected for a dimer of Tec elements. We believe that the slow migrating band in lane 1 is a dimer circle. We have identified several examples of Tec elements inserted into Tec elements (Krikau and Jahn 1991), which could give rise to the dimer circles. (B) Total DNA was reacted with Bal 31 for 0 min (Bal 31 buffer only), 1 min., and $5 \mathrm{~min}$. The DNA was electophoresed with or without prior BamHI restriction digestion, Southern blotted, and probed with a 2.9-kb EcoRI fragment Tecl junction clone. Bal 31 treatment linearizes the circle, and the subsequent exonuclease activity results in a decrease in the size of the linearized element (lanes 1-3). BamHI digestion after Bal 31 treatment reveals two additional bands, 3.6 and 1.7-kb in size (lane 5). Continued exposure to Bal 31 results in a decrease in size for both of these fragments (lane 6), indicating that the exonuclease activity of Bal 31 is able to proceed in both directions after linearization. These bands correspond to the linearization occurring at the junction of the inverted repeats.

zymes and then treating with S1 nuclease. Digesting the elements with restriction enzyme would release the torsional stress. This gave identical results to the reverse order; thus, the sensitivity is not dependent on the circular structure. In addition, any unusual DNA conformation would have to be present at a $\mathrm{pH}$ range of 5.0-8.0 to be reactive with all three nucleases. Taken together, these results suggest that the nuclease reactivity is not the result of a non-B DNA conformation. Finally, we have tested whether the nucleases would react at sites of heteroduplex DNA. Two clones of the excised D7 IES circle junctions derived from different micronuclear alleles were obtained from Klobutcher's laboratory (Baird et al. 1989; Tausta and Klobutcher 1990; Tausta et al. 1991). Denaturation and renaturation of fragments from the two clones yielded 474-bp fragments that contained a heteroduplex region consisting of a single mismatch and 5 mismatched bases surrounding 9 paired bases. The heteroduplex region occurs 292 bp from one end and 185 bp from the other; thus, cutting at the heteroduplex produces fragments of these sizes. Under our nuclease reaction conditions, all three nucleases react at this region, confirming the detection of at least five base pair heteroduplexes (data not shown).

\section{Alkaline denaturing electrophoresis}

To determine whether a gap exists in one strand at the junction, we digested gel-purified circular forms of Tec (see Materials and methods) with restriction enzymes and analyzed the digestion products on denaturing $(\mathrm{NaOH})$ agarose gels that were Southern blotted and hybridized with Tecl or Tec2 junction probes. We would expect to see single-stranded fragments of the sizes produced by $\mathrm{S} 1$ or Bal 31 cleavage if a specific gap were at the junction. Shown in Figure 5 is undigested and BamHI- or EcoRI-digested isolated Tec circles electrophoresed in either a TAE-buffered agarose gel (lanes 1-3) or an alkaline-denaturing gel (lanes 4-6). The Southern blots were hybridized with a Tecl-specific probe. The circles migrate slower than the linearized BamHI-digested circles (lane 1 vs. 2), indicating that gel purification has not affected the mobility of the circles. However, during alkaline electrophoresis, the circles migrate as single-stranded linear molecules (lane 4 vs. 5). In BamHI-digested circular DNA electrophoresed in an alkaline gel (lane 5), a single linear band is present. The absence of the 3.6- and 1.7-kb BamHI fragments visible after nuclease digestion indicates that the circles are not nicked or gapped at the junction of the inverted repeats. The EcoRI digestion pattern supports this conclusion further because only the characteristic $2.9-\mathrm{kb}$ junction band and polymorphic background bands are visible after alkaline electrophoresis. The identical results were obtained when Southern blots of HindIII and $\mathrm{XbaI}$ digests of circular Tec run in TAE versus alkaline gels were hybridized with Tec2-specific probes (not shown).

From the above results we conclude that the relaxed circles present in most of our DNA preparations are the result of random nicks and that a nick or gap does not 


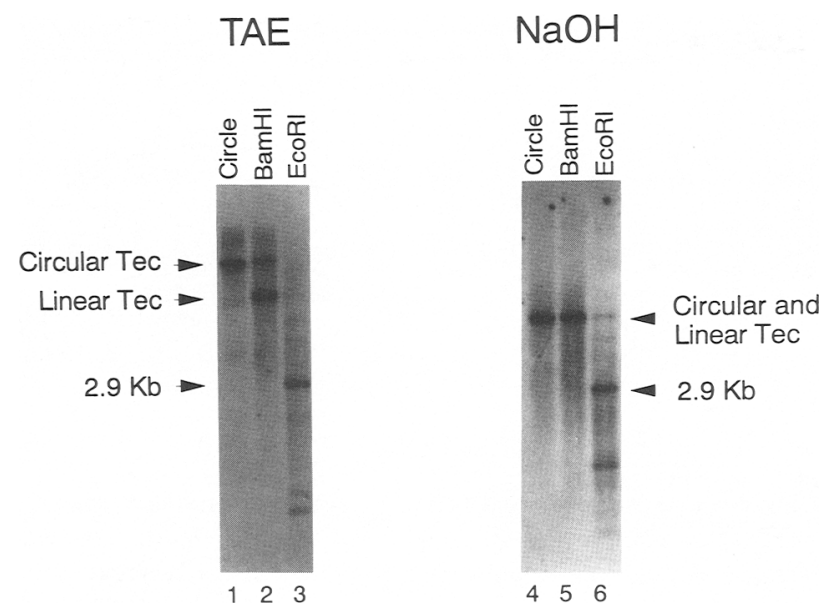

Figure 5. Alkaline electrophoresis. Denaturing $\mathrm{NaOH}$ electrophoresis was used to determine whether a specific nick or gap exists at the junction of excised circular Tec1. Gel-purified circle (see Materials and methods), either native or restriction enzyme-digested, was electrophoresed through either a TAE- or $\mathrm{NaOH}$-buffered agarose gel, Southern blotted, and probed with a 2.9-kb EcoRI fragment junction clone specific for Tecl. In the TAE gel, the circle is still intact after gel purification (lane 1) and is partially linearized by $\mathrm{BamHI}$ (incomplete digest) (lane 2). $E c o$ RI digestion produces the characteristic $2.9-\mathrm{kb}$ fragment (lane 3). Denaturing electrophoresis results in the circle migrating at the size of the linear excised Tec (lane 4). This could be the result of the presence of single random nicks or comigration of circular and linear forms in this gel system. Both BamHI and EcoRI-digested circular DNA produce the same size fragments in the $\mathrm{NaOH}$ gel (lanes 5,6), as observed in the TAE gel. Most importantly, there are no additional bands present in either lane. If a nick or gap was present at the junction, we would expect to see the additional bands corresponding in size to those present in the nuclease experiments. The denaturation of the DNA in the $\mathrm{NaOH}$ gel was confirmed by comparing a randomly nicked restriction-digested plasmid run through the TAE and $\mathrm{NaOH}$ gels. In the TAE gel, all of the fragments were visible; in the $\mathrm{NaOH}$ gel, the digest produced a smear (data not shown).

exist specifically at the junction. This leaves the possibility of a heteroduplex. We would expect that if heteroduplex occurred at the junction, cloning of the junction could give rise to two different plasmids upon replication of the clone; thus, we have attempted to find two sequence alternatives in pools of clones derived from a single cloned junction sequence. For two cloned junctions, we have sequenced eight isolates from the original clone and found all to be identical. In both cases, an oligonucleotide corresponding to the junction sequence was used as a hybridization probe back to filter lifts of $\sim 300$ colonies grown from the original clone. In both cases, all of the colonies hybridized to the sequence, indicating that no sequence heterogeneity exists in these cloned junctions. This is not surprising for the following two reasons. First, the junction could undergo mismatch repair in E. coli. Second, the 700-bp inverted repeats are unstable in $E$. coli, and very few junctions are recovered; thus, the stability in $E$. coli may be very sensitive to the exact sequence at the junction, causing selective loss of one of the two products. We may need to try this analysis on many more junction clones. Ultimately, a DNA transformation system or in vitro system using defined substrates will allow us to address the structure more definitively.

\section{Discussion}

Sequence analysis of Tec element termini and characterization of the circular excision products have yielded data that are critical to our understanding of the mechanism of excision. The sequence analysis shows high conservation of a sequence motif that is thought to be involved in chromosome fragmentation. This chromosome fragmentation consensus was established as follows. First, the sequence analysis of macronuclear telomeres using Maxam-Gilbert sequencing of total macronuclear DNA revealed a readable sequence of the telomeric $\mathrm{C}_{4} \mathrm{~A}_{4}$ repeats, followed by $17 \mathrm{bp}$ of randomized sequence that was followed by 5 bp of readable sequence consisting of $5^{\prime}$-TTGAA-3' (Klobutcher et al. 1981). Thus, a large number of macronuclear chromosomes must carry this sequence exactly $17 \mathrm{bp}$ internal to the telomere (illustrated in Fig. 1B). Second, analysis of the micronuclear precursor sequences for several macronuclear molecules showed that this sequence can also reside in the flanking micronuclear sequences (Baird and Klobutcher 1989). In this case, the TTGAA is on the opposite strand at a position $11 \mathrm{bp}$ from the site of telomere addition. Alignment of micronuclear and macronuclear sequences led to the consensus shown in Figure $1 \mathrm{~B}$, with a conserved location $10 \mathrm{bp}$ downstream or $4 \mathrm{bp}$ upstream of the telomere addition site. The consensus sequence in the Tec element termini is similar, but identical for only TTGAA, which is considered to be the conserved "core" of the fragmentation consensus. Most importantly, this core in the Tec element termini has similar spacing relative to the end of the element (Fig. 1B). Thus, if the location of this core controls the site of cleavage for either formation of a macronuclear chromosome or Tec excision, we would expect a cleavage at the end of the Tec elements within the TA target site duplication. However, the results of characterization of Tec circular form junctions described here implicate a different cleavage from that predicted for chromosome fragmentation. In chromosome fragmentation, the cleavage site $17 \mathrm{bp} 5^{\prime}$ to TTGAA is predicted to form the $5^{\prime}$ end of a staggered cleavage (Baird and Klobutcher 1989). As discussed below, our model of Tec circle formation predicts an opposite orientation of the staggered cut, where the cut made $17 \mathrm{bp}$ away from the TTGAA would form the $3^{\prime}$ end.

Characterization of Tec element circular excision products has revealed structural features that implicate a specific excision mechanism. We report here that the inverted repeat junctions of excised circular Tec elements contain 10 extra bases between both target site duplications. These extra 10 bases share no obvious sequence or structural similarity with each other. Dena- 
turing electrophoresis demonstrated that the junction does not contain a nick or gap and therefore must be closed. The junction is endonucleolytically cleaved by the nucleases S1, mung bean, and Bal 31. Furthermore, this cleavage does not appear to be the result of any secondary structure inherent in DNA circles containing the inverted repeats, as the nuclease sensitivity persists when the circles are restriction enzyme digested. This suggests that heteroduplex DNA may exist at the junction of the inverted repeats in Tec circles. As described in the introductory section, precedence for heteroduplex at the junction of an excised circular transposon comes from Tn916 (Caparon and Scott 1989).

In developing a model of the mechanism of excision of Tec elements, three important facts have been taken into consideration. First, Tec excision appears to be a precise process, leaving a single copy of the target site duplication at the micronuclear location from which it originated (Baird et al. 1989; Tausta et al. 1991; Krikau and Jahn 1991). However, the excised circle contains two copies of the target site duplication. Therefore, the excision must involve staggered cuts and some DNA synthesis. Second, Tec circles contain 10 extra bp between the target site duplications of the junction. These extra bases presumably originate from the micronuclear region immediately adjacent to each Tec element. Although this cannot be established directly, it seems unlikely that a specific number of random bases would be added in a template-independent fashion. Finally, we must account for the nuclease sensitivity of the junctions. The model presented below allows for the presence of heteroduplex DNA at the junction.

We envision the excision beginning with a staggered cut at each end of the element within the target site duplication on one strand and on the opposite strand 11 bases away (see Fig. 6). This would generate two 11-bp 5' extensions. To form the circle, the outside 10 bases of each extension are held together by the protein complex without nucleotide pairing but appropriately to allow the single-base gap on each side to be properly filled in and ligated to the $5^{\prime}$ ends. This single-base addition will recreate both target site duplications between the inverted repeats, and the sealing of the circle will create a 10-base heteroduplex at the junction. The parent chromosome will be repaired by filling in the ends and bluntend joining to recreate the single TA at the original site of the Tec element. This model can be modified to produce heteroduplex DNA 2-10 bp in length if the original staggered cut varies from 7 to 11 bases in length. For example, if the original staggered cut creates a 7-base extension and the protein complex holds 2 bases of heteroduplex together, then 5 bases would be filled in on each end before sealing the circle. If the length of the extension is not equal on both sides, even more possibilities exist. In addition, the cleavage could occur at the ends of the element instead of inside the target site duplication: This would result in a 2-bp overlap (TA) of the chromosomal ends with fill-in and ligation (Fig. 6, rejoined Mic sequences) and fill-in of the target site duplications on the Tec circular junction.

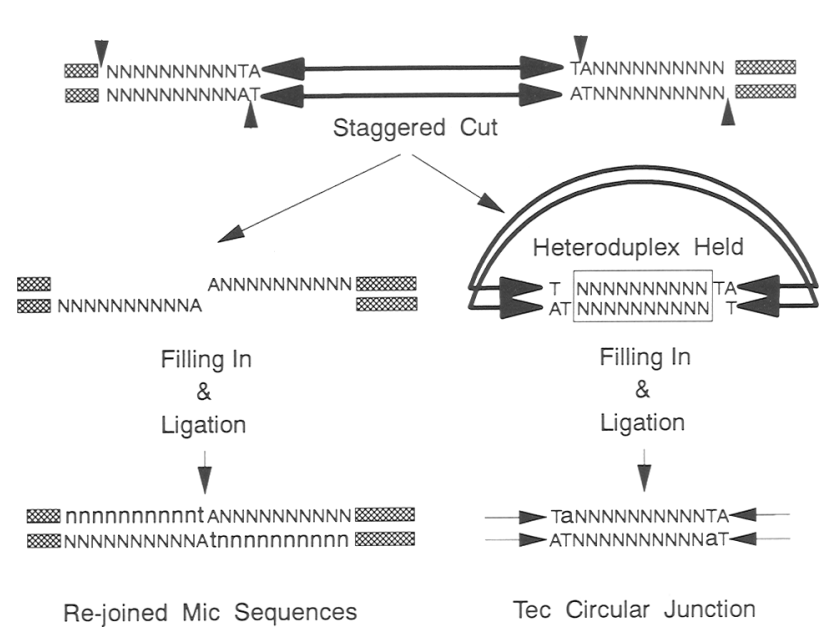

Figure 6. Model for Tec excision. (Top) Tec integrated within the micronucleus. Tec inverted repeats are represented by the arrows. The TA located outside each inverted repeat is the target site duplication, and the N's are the next 10 bases outside the element. The crosshatched boxes represent adjacent micronuclear sequences. Excision begins with a staggered cut, where one cut is within the target site duplication and the other is outside the element generating an 11-base 5' extension from the sequences flanking each end of the element. At this point, the excised element and adjacent micronuclear sequences are processed differently. The outer 10 bases of the 5 ' extension of the element is then held together without nucleotide pairing (boxed sequences) such that the inverted repeats are now in a head-tohead orientation. The single nucleotide gap is then filled in with DNA polymerase and sealed with DNA ligase, resulting in heteroduplex DNA located at the junction. The final junction contains the inverted repeats joined in a head-to-head orientation and both target site duplications surrounding 10 extra bases. The adjacent micronuclear sequences are simply filled in using DNA polymerase and blunt-end joined using DNA ligase. This results in a single TA present at the site from which Tec excised. This model can be varied by decreasing both the length of the $5^{\prime}$ extension and the number of bases held together as a heteroduplex.

This model is consistent with the known facts involving the excision of Tec and is supported further by the model of $8+2$ IES excision presented by Klobutcher et al. (this issue). They have sequenced the circular junctions of the V1 and V3 IESs and compared that sequence to the bases immediately adjacent to the IESs in the micronuclear genome. They determined that the junction was composed of 10 extra bases inserted between both copies of the TA terminal direct repeat. Furthermore, 8 bases were from one side of the IES and 2 bases were from the opposite side. The IES excision model was developed by analyzing the junction sequences of the two excised circular IESs using a strand-biased polymerase chain reaction (PCR) approach. This approach suggests the presence of a 6-base heteroduplex at the center of the junction and allowed Klobutcher et al. to present a model involving staggered cuts, where one strand is cut at the target site duplication and the other is cut 10 bases away resulting in a 10-base 5 ' extension. The sequence of 
one of the junctions that we have cloned (Fig. 1C, pJ12) is unusual and interesting with respect to the above $8+2$ model. The first 8 bases of this junction perfectly match the first 8 bases of the consensus Tec inverted repeat. This could result from two Tec elements being integrated adjacent to one another with a single TA separating them. If the total length of $5^{\prime}$ extension for Tec excision is 9 bases (see Fig. 6), we would expect 6 bases of heteroduplex and the resulting circle junction to contain a $8+2$ arrangement as seen in this junction and the IES circular junctions.

The above discussion points to relationships between Tec excision and both IES excision and chromosome fragmentation. Although the TTGAA core sequence was originally thought to be a chromosome fragmentation consensus, it clearly has a broader role and is not the sole determinant of cleavage of the micronuclear chromosome. Thus, a protein interacting with this site may protect the DNA in a manner that directs cleavage to a site $17 \mathrm{bp}$ away from the core. The specific cleavage would be carried out by different enzymes for Tec excision and fragmentation, leading to two types of staggered ends. The above models suggest that IESs would utilize the same cleavage enzymes as Tecs. However, because IESs do not bear the TTGAA core, some other mechanism must be involved in the recognition of their ends for cleavage. A requirement for different recognition factors in IES excision and for different cleavage factors in chromosome fragmentation could explain the differences in the developmental timing of all of these processes.

Given the relationship between Tec element termini and the chromosome fragmentation core consensus, it is unclear why circular Tec elements are not linearized and subjected to telomere addition. Chromosome fragmentation occurs $30-40 \mathrm{hr}$ after Tec excision, whereas Tec circular forms are stable up to $48 \mathrm{hr}$ postexcision. One possibility is that the circle junction is completely inaccessible to the fragmentation machinery either because of its circular structure (no available ends) or because of the head-to-head association of the inverted repeats, which would bring together multiple TTGAA sites.

The question remains as to whether Tec excision is related to transposition. We have recently identified Tec element transcripts present during macronuclear development (J. Jaraczewski, unpubl.). Thus, element-encoded functions could play a role in excision. To date, Tn916 is the only transposon known to involve an excised covalently closed circular transposition intermediate (Scott et al. 1988). Although it has provided us with a model for the structure of the Tec circle junctions, Tn916 is clearly unusual as a transposon; its structure differs (imperfect terminal inverted repeat sequences and no target site duplication). In addition, the element genes involved in transposition are related to lambdoid phage Int and Xis functions (Poyart-Salmeron et al. 1989, 1990). Thus, transposition in this case could be characterized more as a site-specific rearrangement process. The nature of DNA elimination in hypotrichous ciliates, that is, the requirement for rearrangement in the production of functional macronuclear genes, suggests that the cir- cular products should be nonreactive and, hence, would not serve as a transposition intermediate. In this respect, the circular IES and Tec products resemble the circular products of immunoglobulin or T-cell receptor gene rearrangement, that is, they are being thrown away. Further analysis of the sequence requirements for Tec excision and the function of $\mathrm{Tec}$ element gene products should allow us to distinguish between transposition and excision.

\section{Materials and methods \\ Cell culture and DNA preparation}

E. crassus strains 8 and 24 were cultured and harvested as described in Krikau and Jahn (1991). Total DNA was prepared from mated cells by treatment with proteinase $\mathrm{K}$ followed by phenol extraction and ethanol precipitation as described in Jahn et al. (1989). The DNA precipitate was immediately centrifuged after the addition of ethanol to reduce the amount of carbohydrate contamination. Anlagen were isolated as described in Roth et al. (1985), and the DNA was purified as described above.

\section{Cloning and sequencing Tec1 and Tec2 junctions}

Tecl junctions contained within 2.9-kb EcoRI fragments were cloned by first gel-purifying circular Tec from anlagen DNA electrophoresed in TAE buffer $(40 \mathrm{mM}$ Tris-acetate, $1 \mathrm{mM}$ EDTA at $\mathrm{pH} 8.0$ ) using NA45 paper (Schleicher \& Schuell) according to the manufacturer's suggested protocol. Purification of anlagen results in nicking of the Tec circular forms, and only relaxed circles and linears are found in the anlagen DNA samples. Thus, the eluted circles were always relaxed circular forms. EcoRI restriction-digested Tec circular DNA was cloned into the $\lambda$ phage vector gt10 (Huynh et al. 1985). Approximately 2000 phage were plated, plaque lifted in triplicate, and hybridized with one of the following: (1) an inverted repeat specific probe; (2) a probe from a region within the $2.9-\mathrm{kb}$ junction fragment but outside the inverted repeat; or (3) a probe from a region of the element completely outside the $2.9-\mathrm{kb}$ junction fragment. Forty phage were selected that hybridized only to probes specific for the $2.9-\mathrm{kb}$ junction fragment. Two of these, $\mathrm{pJ} 8$ and pJ34, contained a 2.9-kb EcoRI fragment. The pJ8 junction was subcloned further using a polymorphic PstI site located $250 \mathrm{bp}$ from the junction. pJ34 was subcloned further as a 400 -bp AluI fragment using AluI sites located $200 \mathrm{bp}$ from the junction.

Tecl junctions contained within 1.1-kb Pst Iragments were subcloned by restriction digestion of gel-purified circle with PstI and cloning into the plasmid vector pKS + (Stratagene). The clones were then transformed into the E. coli strain SURE (Stratagene). From four independent ligations and transformations, $>1000$ individual colonies were picked onto new plates, colony lifted, and hybridized with a probe specific for the inverted repeats. Three clones contained $1.1-\mathrm{kb}$ junction fragments: $\mathrm{p} 2$, pJ12, and pJ46. $\mathrm{p} 2$ was subcloned further using a single HindIII site located 125 bases from the junction. pJ12 and pJ46 were subcloned as nested deletions using the Erase-A-Base system (Promega).

Tec2 junctions contained within 2.5-kb HindIII-EcoRI fragments were cloned into the vector $\mathrm{pKS}+$ and transformed into SURE. $\sim 500$ colonies were picked onto fresh plates, colony lifted, and probed with an oligonucleotide specific for Tec2 inverted repeats. Four clones contained a $2.5-\mathrm{kb}$ junction: pJ4, pJ6, $\mathrm{pJ} 7$, and pJ9. Both pJ4 and $\mathrm{pJ} 7$ junctions were subcloned further as 310-bp NlaIII fragments into the SphI site in pBS (Stratagene). 
Both pJ6 and pJ9 were too unstable, even in SURE, to be subcloned further or to be sequenced at the junction.

To purify supercoiled Tec circles for direct sequence analysis of the junctions, total DNA (1 mg) isolated from cells at various points of macronuclear development was centrifuged in a CsCl/ ethidium bromide gradient at $40 \mathrm{~K}$ for $48 \mathrm{hr}$ in a Ti50 rotor (Beckman), as described for the large-scale purification of plasmid DNA (Maniatis et al. 1982). The gradients were fractionated in $0.5-\mathrm{ml}$ aliquots, and $10 \mu \mathrm{l}$ from each aliquot was loaded directly into an agarose gel, electrophoresed, Southern blotted, and hybridized with a Tecl-specific probe. Aliquots containing supercoiled Tec were identified by the presence of a hybridizing band migrating at $\sim 4.0-\mathrm{kb}$ in size. Fractions from four gradients were pooled to obtain enough supercoiled Tec DNA for one sequencing reaction.

Double-stranded sequencing through the inverted repeat junction of clones or purified supercoiled Tec circles was performed using Sequenase essentially as described in Kraft et al (1988).

\section{Nuclease experiments}

Total DNA from various time points of macronuclear development was exposed to $\mathrm{S} 1$ nuclease at $27 \mathrm{U} / \mu \mathrm{g}$ of DNA at $30^{\circ} \mathrm{C}$ in $0.05 \mathrm{M} \mathrm{NaOAc}$ (pH 5.7), $0.2 \mathrm{M} \mathrm{NaCl}, 1.0 \mathrm{mM} \mathrm{ZnSO}_{4}, 0.5 \%$ glycerol. The reaction was terminated by bringing the final volume to $0.1 \mathrm{M}$ Tris- $\mathrm{HCl}(\mathrm{pH} 7.6)$ and $5 \mathrm{~mm}$ EDTA and transferring to $4^{\circ} \mathrm{C}$. Bal 31 was used at $0.2 \mathrm{U} / \mu \mathrm{g}$ of DNA at $30^{\circ} \mathrm{C}$ in $12 \mathrm{~mm}$ $\mathrm{CaCl}_{2}, 12 \mathrm{mM} \mathrm{MgCl}_{2}, 0.6 \mathrm{M} \mathrm{NaCl}, 20 \mathrm{~mm}$ Tris- $\mathrm{HCl}$ (pH 8.0), and $1 \mathrm{mM}$ EDTA, and the reaction was stopped by adding EGTA to $50 \mathrm{mM}$ and transferring to $4^{\circ} \mathrm{C}$. Mung bean nuclease was used at $15 \mathrm{U} / \mu \mathrm{g}$ of DNA at $30^{\circ} \mathrm{C}$ in $30 \mathrm{~mm} \mathrm{NaOAc}(\mathrm{pH} 5.0), 100 \mathrm{~mm}$ $\mathrm{NaCl}, 2.0 \mathrm{mM} \mathrm{ZnCl}_{2}, 10 \%$ glycerol and the reaction was terminated by increasing the $\mathrm{NaCl}$ concentration to $0.2 \mathrm{M}$ at $4^{\circ} \mathrm{C}$. All of the above reactions were extracted with phenol and phenolchloroform before ethanol precipitation. After a $70 \%$ ethanol wash, the DNA was dried and resuspended in TE (10 mM Tris$\mathrm{HCl}$ at $\mathrm{pH} 7.5,1 \mathrm{~mm}$ EDTA). The restriction enzyme digestions, agarose gel electrophoresis, Southern blotting, DNA-labeling reactions, and hybridizations were carried out by standard procedures as described previously in Krikau and Jahn (1991).

\section{Alkaline electrophoresis}

Circular Tec was gel purified from anlagen DNA using USBioclean (U.S. Biochemical) according to the manufacturer's suggested protocol. Because we are searching for nicks in the DNA, we did not want the DNA to be stained with ethidium bromide or exposed to UV light; therefore, an extra lane was included during the electrophoresis, which was subsequently stained and visualized. By measuring the distance that the circle migrated in the extra lane, we were able to purify the circles from the other lanes without exposing them to either ethidium bromide or UV light.

The alkaline gel was prepared by making a $1 \%$ agarose gel containing $50 \mathrm{mM} \mathrm{NaCl}$ and $1 \mathrm{~mm}$ EDTA. The gel was then equilibrated overnight using $30 \mathrm{~mm} \mathrm{NaOH}, 1 \mathrm{~mm}$ EDTA (Maniatis et al. 1982). Electrophoresis was performed at $3 \mathrm{~V} / \mathrm{cm}$ in a cold room at $4^{\circ} \mathrm{C}$. The buffer was recirculated during electrophoresis. A glass plate was placed on top of the gel to ensure that the gel did not float during the run. Before loading, the DNA was denatured in $50 \%$ formamide, $10 \mathrm{mM}$ EDTA, at $85^{\circ} \mathrm{C}$ for 5 min and snap cooled.

\section{Acknowledgments}

We thank L. Klobutcher for discussion of results before publi- cation. This work was supported by U.S. Public Health Service grant GM37661 to C.L.J. from the National Institutes of Health.

The publication costs of this article were defrayed in part by payment of page charges. This article must therefore be hereby marked "advertisement" in accordance with 18 USC section 1734 solely to indicate this fact.

\section{References}

Baird, S.E. and L.A. Klobutcher. 1989. Characterization of chromosome fragmentation in two protozoans and identification of a candidate fragmentation sequence in Euplotes crassus. Genes \& Dev. 3: 585-597.

Baird, S.E., G.M. Fino, S.L. Tausta, and L.A. Klobutcher. 1989. Micronuclear genome organization in Euplotes crassus: A transposonlike element is removed during macronuclear development. Mol. Cell. Biol. 9: 3793-3807.

Caparon, M.G. and J.R. Scott. 1989. Excision and insertion of the conjugative transposon $\mathrm{Tn} 916$ involves a novel mechanism. Cell 59: 1027-1034.

Collins, J., G. Volckaert, and P. Neuers. 1982. Precise and nearly precise excision of the symmetrical inverted repeats of Tn5; common features of recA-independent events in Escherichia coli. Gene 19: 139-146.

Devereux, J., P. Haeberli, and O. Smithies. 1984. A comprehensive set of sequence analysis programs for the VAX. Nucleic Acids Res. 12: 387-395.

Doseff, A., R. Martienssen, and V. Sundaresan. 1991. Somatic excision of the $M u 1$ transposable element of maize. Nucleic Acids Res. 19: 579-584.

Fujimoto, S. and H. Yamagishi. 1987. Isolation of an excision product of T-cell receptor alpha-chain gene rearrangements. Nature 327: 242-243.

Gray, H.B. Jr., D.A. Ostrander, J.L. Hodnett, R.J. Legerski, and D.L. Robberson. 1975. Extracellular nucleases of Pseudomonas Bal31. I. Characterization of single-strand specific deoxyriboendonuclease and double-strand deoxyriboexonuclease activities. Nucleic Acids Res. 2: 1459-1492.

Haniford, D.B., H.W. Benjamin, and N. Kleckner. 1991. Kinetic and structural analysis of a cleaved donor intermediate and a strand transfer intermediate in Tn10 transposition. Cell 64: 171-179.

Huynh, T.V., R.A. Young, and R.W. Davis. 1985. Constructing and screening cDNA libraries in lambda gt10 and lambda gtll. In DNA cloning: A practical approach (ed. D.M. Glover|, vol.1, pp. 49-78. IRL Press, Oxford, England.

Jahn, C.L. 1991. Nuclear duality in the hypotrichous ciliates: Maintaining the maximum and the minimum of information. J. Protozool. 38: 252-258.

Jahn, C.L., L.A. Nilles, and M.F. Krikau. 1988. Organization of the Euplotes crassus micronuclear genome. I. Protozool. 35: 590-601.

Jahn, C.L., M.F. Krikau, and S. Shyman. 1989. Developmentally coordinated en masse excision of a highly repetitive element in E. crassus. Cell 59: 1009-1018.

Klobutcher, L.A. and C.L. Jahn. 1991. Developmentally controlled genomic rearrangements in ciliated protozoa. Curr. Opin. Genet. Dev. 1: 397-403.

Klobutcher, L.A. and D.M. Prescott. 1987. The special case of the hypotrichs. In The molecular biology of ciliated protozoa (ed. J. Gall), pp. 111-154. Academic Press, New York.

Klobutcher, L.A., M.T. Swanton, P. Donini, and D.M. Prescott. 1981. All gene-sized DNA molecules in four species of hypotrichs have the same terminal sequence and an unusual $3^{\prime}$ terminus. Proc. Natl. Acad. Sci. 78: 3015-3019. 
Kraft, R., J. Tardiff, K.S. Krauter, and L.A. Leinwand. 1988. Using mini-prep plasmid DNA for sequencing double stranded templates with Sequenase. BioTechniques 6: 544-547.

Krikau, M.F. and C.L. Jahn. 1991. Tec2: A second transposonlike element demonstrating developmentally coordinated excision in Euplotes crassus. Mol. Cell. Biol. 11: 4751-4759.

Kroeker, W.D. and D. Kowalski. 1978. Gene-sized pieces produced by digestion of linear duplex DNA with Mung Bean nuclease. Biochemistry 17: 3236-3243.

Lilley, D.M. 1980. The inverted repeat as a recognizable structural feature in supercoiled molecules. Proc. Natl. Acad. Sci. 77: 6468-6472.

Maniatis T., E.F. Fritsch, and J. Sambrook. 1982. Molecular cloning: A laboratory manual. Cold Spring Harbor Laboratory, Cold Spring Harbor, New York.

McCormack, W.T., L.W. Tjoelker, L.M. Carlson, B. Petryniak, C.F. Barth, E. H. Humphries, and C.B. Thompson. 1989. Chicken IgL gene rearrangement involves deletion of a circular episome and addition of single nonrandom nucleotides to both coding segments. Cell 56: 785-791.

Mizuuchi, K. 1992. Transpositional recombination: Mechanistic insights from studies of $\mathrm{Mu}$ and other elements. Annu. Rev. Biochem. 61: 1011-1051.

Moerman, D.G. and R.H. Waterston. 1989. Mobile elements in Caenorhabditis elegans and other nematodes. In Mobile DNA (ed. D.E. Berg and M.M. Howe), pp. 537-556. American Society for Microbiology, Washington, D.C.

Morisato, D. and N. Kleckner. 1987. Tn10 tranposition and circle formation in vitro. Cell 51: 101-111.

Okazaki, K., D.D. Davis, and H. Sakano. 1987. T-cell receptor beta gene sequences in the circular DNA of thymocyte nuclei: Direct evidence for the intramolecular DNA deletion model in V-D-J joining. Cell 49: 477-485.

Poyart-Salmeron, C., P. Trieu-Cuot, C. Carlier, and P. Courvalin. 1989. Molecular characterization of two proteins involved in the excision of the conjugative transposon Tn1545: Homologies with other site-specific recombinases. EMBO I. 8: 2425-2433.

Poyart-Salmeron, C., P. Trieu-Cuot, C. Carlier, and P. Courvalin. 1990. The integration-excision system of the conjugative transposon Tn916 is structurally and functionally related to those of lambdoid phages. Mol. Microbiol. 4: 1513-1521.

Rose, A.M. and T.P. Snutch. 1984. Isolation of the closed circular form of the transposable element Tcl in Caenorhabditis elegans. Nature 311: 485-486.

Roth, M.R., M.-Y. Lin, and D.M. Prescott. 1985. Large scale synchronous mating and the study of macronuclear development in Euplotes crassus. I. Cell Biol. 101: 79-84.

Ruan, K.-S. and S.W. Emmons. 1984. Extrachromosomal copies of transposon $\mathrm{Tcl}$ in the nematode Caenorhabditis elegans. Proc. Natl. Acad. Sci. 81: 4018-4022.

Scott, J.R., P.A. Kirchman, and M.G. Caparon. 1988. An intermediate in transposition of the conjugative transposon Tn916. Proc. NatI. Acad. Sci. 85: 4809-4813.

Shenk, T.E., C. Rhodes, P.W.J. Rigby, and P. Berg. 1975. Biochemical method for mapping mutational alterations in DNA with S1 nuclease: The location of deletions and temperature sensitive mutations in Simian Virus 40. Proc. Natl. Acad. Sci. 72: 989-993.

Sundaresan, V. and M. Freeling. 1987. An extrachromosomal form of the $\mathrm{Mu}$ transposons of maize. Proc. Natl. Acad. Sci. 84: 4924-4928.

Tausta, S.L. and L.A. Klobutcher. 1989. Detection of circular forms of eliminated DNA during macronuclear development in Euplotes crassus. Cell 59: 1019-1026.

1990. Internal eliminated sequences are removed prior to chromosome fragmentation during development in $\mathrm{Eu}$ plotes crassus. Nucleic Acids Res. 18: 845-853.

Tausta, S.L., L.R. Turner, L.K. Buckley, and L.A. Klobutcher. 1991. High fidelity developmental excision of Tecl transposons and internal elminated sequences in Euplotes crassus. Nucleic Acids Res. 19: 3229-3236.

Wiegand, R.C., G.N. Godson, and C.M. Radding. 1975. Specificity of the S1 nuclease from Aspergillus oryzae. J. Biol. Chem. 250: 8848-8855.

Yao, M.-C. 1989. Site specific chromosome breakage and DNA deletion in ciliates. In Mobile DNA (ed. D.E. Berg and M.M. Howe), pp. 715-734. American Society for Microbiology, Washington, D.C. 


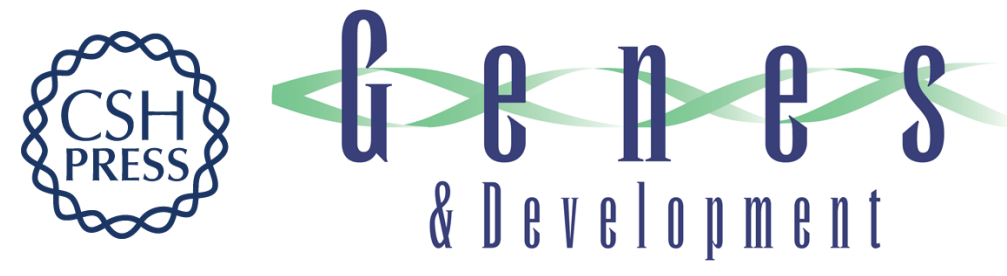

\section{Elimination of Tec elements involves a novel excision process.}

J W Jaraczewski and C L Jahn

Genes Dev. 1993, 7:

Access the most recent version at doi:10.1101/gad.7.1.95

References This article cites 34 articles, 11 of which can be accessed free at: http://genesdev.cshlp.org/content/7/1/95.full.html\#ref-list-1

License

Email Alerting

Receive free email alerts when new articles cite this article - sign up in the box at the Service top right corner of the article or click here.

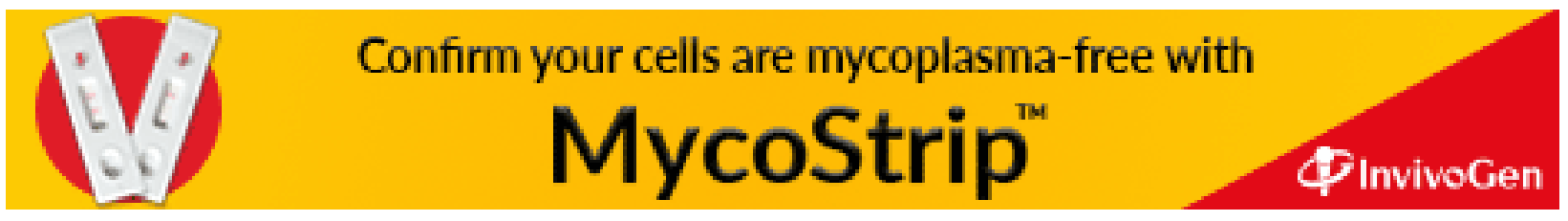

